\title{
Cirugía reparadora de la válvula aórtica bicúspide insuficiente
}

\author{
Ricardo Zalaquett S, Cristóbal Camplá Ca, \\ Maximiliano Scheu $G^{a}$, Samuel Córdova A, \\ Pedro Becker R, Sergio Morán V, Manuel J Irarrázaval LI, \\ Cristian Baeza P, Claudio Arretz V, Sandra Braun J, \\ Gastón Chamorro S, Iván Godoy J, Fernando Yáñez D. \\ Valve repair surgery for incompetent \\ bicuspid aortic valves
}

Background: Surgical valve repair is a good alternative for correction of incompetent bicuspid aortic valve. Aim: To report the early and late surgical, clinical and ecochardiographic results of surgical repair of incompetent bicuspid aortic valves. Patients and methods: Retrospective review of medical records of 18 patients aged 19 to 61 years, with incompetent bicuspid aortic valve in whom a valve repair was performed. Four patients had infectious endocarditis and 17 were in functional class I or II. Follow up ranged from 3 to 113 months after surgery. Results: A triangular resection of the prolapsing larger cusp, which included the middle raphe, was performed in 17 cases; in 13 of these, a complementary subcommisural annuloplasty was performed. In the remaining case, with a perforation of the non-coronary cusp, a pericardial patch was implanted; this procedure was also performed in 2 other cases. In 3 cases large vegetations were removed. Postoperative transesophageal echocardiography showed no regurgitation in 11 patients $(62 \%)$ and mild regurgitation in 7 (38\%). There was no operative morbidity or mortality. There were no deaths during the follow-up period. In 3 patients (17\%) the aortic valve was replaced with a mechanical prosthesis, 8 to 108 months after the first operation. Reoperation was not needed in $93 \% \pm 6,4 \%$ at 1 year and $85 \% \pm 9,5 \%$ at 5 years, these patients were all in functional class I at the end of the follow-up period. $60 \%$ had no aortic regurgitation, $20 \%$ had mild and $20 \%$ moderate aortic regurgitation on echocardiographic examination. A significant reduction of the diastolic diameter of the left ventricle was observed, but there were no significant changes in systolic diameter or shortening fraction. Conclusions: Surgical repair of incompetent bicuspid aortic valves has low operative morbidity and mortality and has a low risk of reoperation (Rev Méd Chile 2005; 133: 279-86).

(Key Words: Aortic valve; Cardiovascular surgical procedures; Heart valve prosthesis implantation)

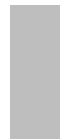

Recibido el 8 de julio, 2004. Aceptado en versión corregida el 21 de diciembre, 2004.

Departamento de Enfermedades Cardiovasculares, Facultad de Medicina, Pontificia Universidad Católica de Chile. Santiago de Chile.

aAyudante Alumno, Escuela de Medicina. Pontificia Universidad Católica de Chile.

$\mathrm{L}^{2}$ a válvula aórtica es una estructura anatómicamente más simple que la válvula mitral, constituida sólo por 3 velos fibromembranosos en forma de "nido de paloma". A diferencia de ésta, que participa directamente en la fisiología ventricular izquierda, la única función de la válvula aórtica es permitir un

Correspondencia a: Dr. Ricardo Zalaquett S. Marcoleta 367, $6^{\circ}$ piso. Teléfonos: 6333030 - 354 3231. Fax: 6390108.

E-mail: rzalaque@med.puc.cl flujo unidireccional de sangre desde el ventrículo izquierdo a la aorta ascendente. Sin embargo, la cirugía reparadora de la insuficiencia aórtica no ha tenido la amplia aceptación que tiene la reparación mitral, que se ha impuesto sobre el reemplazo valvular ${ }^{1-4}$.

En general, la reparación de la insuficiencia valvular aórtica se ha reservado, por la mayoná de los equipos quirúrgicos, para aquellas secundarias a disección o aneurisma de la aorta ascendente 0 a 
comunicación interventricular congénita ${ }^{5-8}$. Situaciones primarias de insuficiencia aórtica susceptibles de reparación senáan la dilatación del anillo valvular aórtico, la válvula aórtica bicúspide, el prolapso de velo en válvula aórtica tricúspide y la perforación de velo aórtico por endocarditis infecciosa ${ }^{6,9-13}$. Otra situación de excepción es la rotura espontánea de un velo a nivel de una comisura asociada a fenestraciones con insuficiencia aórtica aguda ${ }^{14}$.

De todas estas etiologías, en la actualidad la válvula aórtica bicúspide con prolapso del velo mayor y la perforación de velo por endocarditis, son las 2 causas de insuficiencia aórtica primaria más susceptibles de reparación ${ }^{10-13,15,16 .}$.

Recientemente comunicamos nuestra experiencia en cirugía valvular reparadora de la endocarditis infecciosa, incluyendo cierre de perforación de velos de válvulas aórticas tricúspides y bicúspi$\operatorname{des}^{16}$. En esta oportunidad presentamos nuestra experiencia en cirugía reparadora de la válvula aórtica bicúspide insuficiente, incluyendo aquellos pacientes con lesiones endocardíticas asociadas.

\section{PACIENTES Y MÉTODO}

Pacientes. Entre enero de 1994 y abril de 2003, 18 pacientes, todos hombres, portadores de una válvula aórtica bicúspide insuficiente, fueron operados en nuestra institución efectuándoseles una cirugía reparadora de ésta. La edad promedio fue de 40,8 $\pm 12,3$ años, con un margen de 19 a 61 años. Cuatro pacientes tenían una endocarditis sobreagregada. El $71 \%$ de los pacientes se encontraban en capacidad funcional (CF) I o II y el 29\% restante en CF III o IV.

Método. Los pacientes con una cirugía reparadora de una válvula aórtica bicúspide insuficiente fueron identificados a través de la base de datos, específicas de reparaciones valvulares, del Servicio de Cirugía Cardiovascular del Hospital Clínico de la Pontificia Universidad Católica de Chile. Una vez identificados los pacientes, se revisaron las fichas clínicas y protocolos operatorios.

Para el seguimiento clínico, los pacientes, sus familiares 0 médico tratante fueron contactados personal o telefónicamente en julio de 2003, solicitándoseles responder un cuestionario de evaluación clínica. Igualmente, se revisó el último ecocardiograma postoperatorio disponible, el que fue comparado con el preoperatorio en cuanto a grado de insuficiencia aórtica, diámetro sistólico y diastólico y fracción de acortamiento del ventrículo izquierdo.

Técnica quirúrgica. Todos los pacientes fueron operados con circulación extracorpórea con hipotermia sistémica entre 28 y $30^{\circ} \mathrm{C}$, para lo cual se canuló el cayado aórtico y la orejuela derecha con una cánula única dinigida hacia la vena cava inferior, excepto en los casos en que fue necesario acceder a la aurícula izquierda, en los que se canularon ambas venas cavas directamente y por separado con cánulas en ángulo recto y torniquetes elásticos alrededor de ellas. Las cavidades izquierdas fuemon drenadas con un vent aurículo-ventricular introducido a través de la vena pulmonar superior derecha. La protección miocárdica se efectuó con solución cardioplégica cristaloide infundida a $4^{\circ} \mathrm{C}$ por vía retrógrada en todos los casos ${ }^{17}$. Ocasionalmente, la solución cardioplégica fue infundida, además, directamente en los ostia coronarios. La válvula aórtica fue abordada a través de una aortotomía oblicua y expuesta con puntos de sutura supracomisurales.

En los casos en que la insuficiencia aórtica fue secundaria a un prolapso del velo mayor, ésta fue corregida con una resección triangular central de este velo comprendiendo el rafe medio, el que luego fue suturado con TI.CRON ${ }^{\circledR}$ 5-0 ó 6-0 en forma corrida y en 2 planos. Cuando esta reparación fue complementada con una anuloplastia subcomisural, ésta se efectuó con 2 puntos de TI·CRON ${ }^{\circledR} 2-0$ en U vertical apoyados con pledget de teflon felt. Las Figuras 1 a 4 corresponden a fotografías intraoperatorias de uno de estos pacientes.

En los casos con perforación de velos, ésta fue cerrada con un parche de pericardio autólogo previamente fijado en glutaraldehído y suturado con TI·CRON ${ }^{\circledR}$ 5-0 ó 6-0 en forma continua.

En todos los casos la calidad de la reparación valvular aórtica fue evaluada intraoperatoriamente post circulación extracorpórea con un ecocardiograma transesofágico efectuado por un cardiólogo ecocardiografista.

Análisis estadístico. Los datos se expresan como promedio \pm desviación estándar y la sobrevida se analizó mediante el método de Kaplan-Meier. Para la comparación de los parámetros ecocardiográficos pre y postoperatorios se utilizó el test no paramétrico de sumas de rangos de Wilcoxon, 
considerándose significativo un valor $\mathrm{p}<0,05$. Para la presentación de los resultados se siguieron, cuando fue pertinente, la recomendación del comité conjunto de la American Association for Thoracic Surgery y la Society of Thoracic Surgeons ${ }^{18}$.

\section{RESULTADOS}

Hallazgos intraoperatorios y procedimientos quirúrgicos. Todas las válvulas eran bicúspides, con

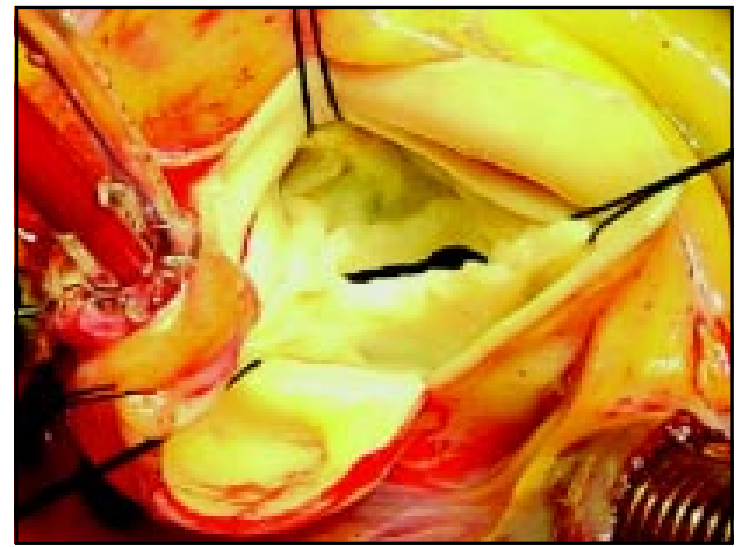

FiguRA 1. Fotografía intraoperatoria de paciente con válvula aórtica bicúspide insuficiente. Los 3 pilares comisurales, incluyendo el correspondiente al rafe medio, están traccionados con puntos de seda. Es posible observar la apertura transversal, en "boca de pescado", de la válvula aórtica (ver texto).

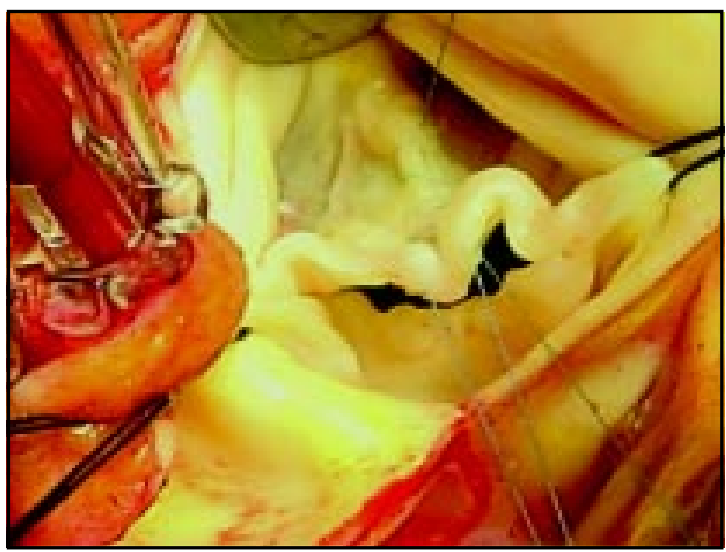

FiguRA 3. Se ha completado la sutura de la resección triangular del velo mayor o fusionado con TI·CRON ${ }^{\circledR}$ $5 / 0$, en forma continua y en 2 planos (ver texto). distintos grados de desarrollo del rafe medio del velo mayor o fusionado. En 4 casos (22\%) existían evidencias de una endocarditis sobreagregada. El prolapso de este velo hacia la cavidad del ventrículo izquierdo fue la principal causa de la insuficiencia aórtica en 17 casos, la que fue corregida con una resección triangular, descrita en Pacientes y Método, la que, a su vez, en 13 casos se complementó con una anuloplastia subcomisural bilateral (Figuras $1 \mathrm{a}$ 4). En el caso restante, la insuficiencia aórtica fue secundaria a una perforación del velo no comnaria-

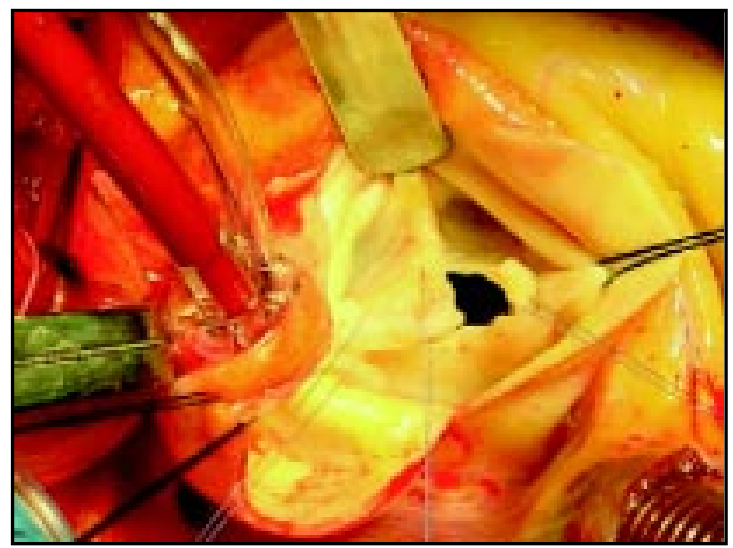

FIGURA 2. Se ha iniciado la resección triangular de la porción media prolapsante del velo mayor o fusionado (ver texto).

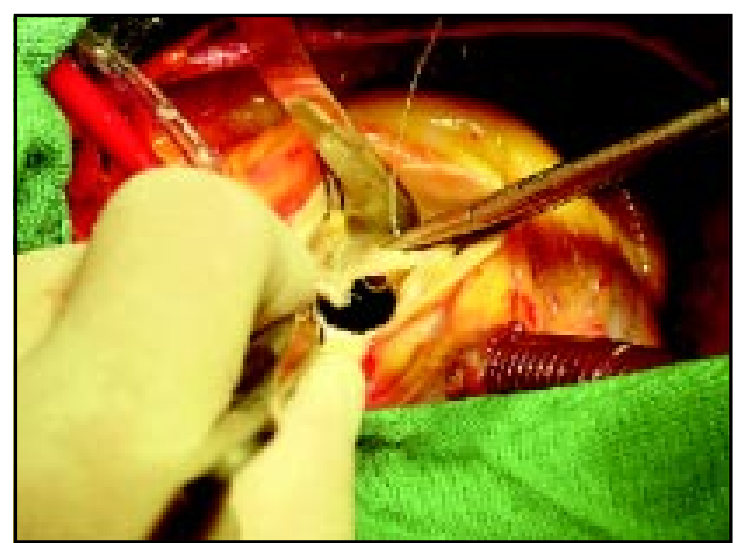

FIGURA 4. La resección triangular del velo mayor o fusionado es complementada con una anuloplastia subcomisural bilateral con 2 puntos de TI.CRON ${ }^{\circledR} 2 / 0$ en $\mathrm{U}$ vertical apoyados con pledget de Teflon felt (ver texto). 
no (no fusionado) por endocarditis infecciosa, la que se presentó, además, en otros 2 casos, y en todos los cuales fue cerrada con un parche de pericardio autólogo. Igualmente, en 3 casos, existían grandes vegetaciones que dificultaban la coaptación de los velos, incrementando la insuficiencia aórtica, las que fueron resecadas. La Tabla 1 resume los procedimientos quirúrgicos efectuados.

La ecocardiografía transesofágica intraoperatoria post reparación demostró ausencia de insuficiencia aórtica en 11 pacientes (62\%) e insuficiencia leve (1+) en 7 (38\%).

En 7 pacientes se efectuó, además, un procedimiento quirúrgico asociado. En 4 de éstos (22\%), éste consistió en una reparación mitral, de acuerdo a principios y técnicas previamente reportados $3,4,19,20$, en uno, a un recambio mitral, en otro, a una revascularización miocárdica y finalmente en otro, a una resección de un mixoma auricular izquierdo.

Morbimortalidad. En esta serie no hubo mortalidad ni morbilidad perioperatoria.

Seguimiento clínico. El seguimiento se cerró en julio de 2003 y se completó en el 100\% de los casos con un promedio de $47,5 \pm 39,9$ meses y un margen de 3 a 113 meses.

Ningún paciente falleció durante el período de seguimiento y solo un paciente fue rehospitalizado por una causa cardiovascular.

Tres pacientes (17\%) requirieron ser reoperados, en promedio a los 44,7 meses (margen: 8 a 108 meses), efectuándose en todos ellos un reemplazo valvular aórtico con prótesis mecánica. Dos de estos pacientes habían tenido originalmente una reparación mitral asociada y el otro una endocarditis aórtica, el que presentó a los 18 meses una nueva endocarditis en el curso de una aplasia medular secundaria al tratamiento antibiótico por la primera endocarditis. Así, la probabilidad de estar libre de reoperación fue de $93 \% \pm 6,4 \%$ a 1 año y $85 \% \pm 9,5 \%$ a 5 años (Figura 5).

Los 15 pacientes no reoperados se encontraban al cierre del seguimiento en CF I. Tres pacientes estaban en tratamiento anticoagulante oral, uno de ellos por ser portador de una prótesis mitral mecánica y los otros 2 por fibrilación auricular.

Seguimiento ecocardiográfico. En todos los pacientes se efectuó un ecocardiograma en algún

\section{Tabla 1. Procedimientos quirúrgicos reconstructores aórticos efectuados en 18 pacientes con válvula aórtica bicúspide insuficiente}

\begin{tabular}{|lcc|}
\hline $\begin{array}{l}\text { Procedimientos } \\
\text { reconstructores }\end{array}$ & $\mathrm{n}^{\circ}$ casos & $\%$ \\
\hline Resección triangular & 17 & 94 \\
Anuloplastia subcomisural & 13 & 72 \\
Parche perforación & 3 & 17 \\
Resección vegetaciones & 3 & 17 \\
\hline
\end{tabular}

momento luego del alta hospitalaria. Para los pacientes no reoperados éste se efectuó en promedio a los $33 \pm 40$ meses con margen de 2 a 104 meses. La Tabla 2 resume el grado de insuficiencia aórtica postoperatoria para estos 15 pacientes. El $60 \%$ de ellos no tenía insuficiencia aórtica y el $20 \%$ presentaba una insuficiencia leve (1+).

La Tabla 3 compara los diámetros ecocardiográficos diastólicos y sistólicos así como la fracción de acortamiento del ventrículo izquierdo pre y postoperatoria en estos mismos pacientes, observándose una disminución estadísticamente significativa en el diámetro diastólico pero no así en los otros parámetros.

\section{DISCUSIÓN}

La válvula aórtica bicúspide es una malformación congénita que se presenta en aproximadamente el $2 \%$ de la población ${ }^{21,22}$. De éstos, más de $60 \%$ no presentará disfunción valvular durante toda la vida $^{23}$. La regurgitación aórtica como complicación de una válvula bicúspide se presenta más frecuentemente en pacientes jóvenes, como en esta experiencia ${ }^{21,22}$. Por otra parte, mientras más jóvenes los pacientes, más factible es reparar la válvula puesto que es menos probable que esté calcificada o fibrosada. Por lo mismo, la mayoría de los pacientes estaban asintomáticos o poco sintomáticos en esta experiencia, como en otras de la literatura ${ }^{10-13}$. Lo anterior también es resultado de la evidencia más reciente que ha demostrado que una estrategia conservadora de la insuficiencia aórtica esperando crecimiento o disfunción ventricular izquierda o síntomas para indicar la cirugía, se traduce en una menor expectativa de vida ${ }^{24}$. 


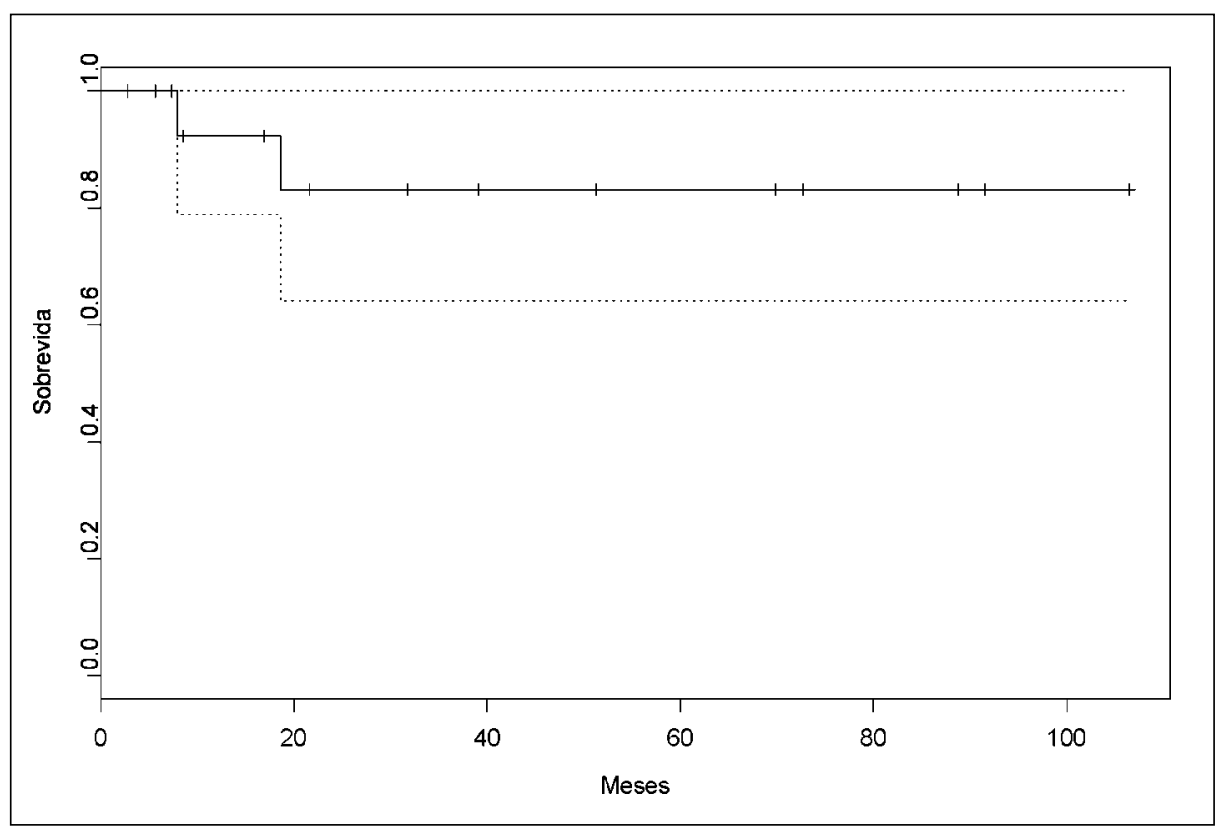

FiguRA 5. Curva de probabilidad actuarial (Kaplan-Meier) de estar libre de reoperación para pacientes con cirugía reparadora de válvula aórtica bicúspide insuficiente.

Tabla 2. G rado de insuficiencia aórtica ecocardiográfica postoperatoria alejada en 15 pacientes con cirugía reparadora de válvula aórtica bicúspide insuficiente

\begin{tabular}{|lcc|}
\hline Insuficiencia aórtica & $\mathrm{n}^{\circ}$ casos & $\%$ \\
\hline Sin & 9 & 60 \\
Leve $(+)$ & 3 & 20 \\
Moderada (++) & 3 & 20 \\
\hline
\end{tabular}

Hallazgos intraoperatorios y procedimientos quirúrgicos. La válvula bicúspide es el resultado de la fusión de los velos de los senos de Valsalva coronarios derecho e izquierdo y de aquí que en relación a este velo fusionado y muy cerca de las comisuras estén ambos ostia coronarios, uno en posición más anterior (derecho) y el otro en posición más posterior (izquierdo). Por lo mismo, en la mayoría de los casos es posible observar un rafe medio en el velo fusionado, de mayor o menor desarrollo, que se origina de una cúspide fibrosa $^{25}$. Es esto lo que crea cierta confusión entre los cirujanos y ecocardiografistas, ya que estrictamente estas válvulas son tricúspides pero bicomisurales, y llamarlas por este último término

Tabla 3. Parámetros ecocardiográficos pre y post reparación aórtica alejados en 15 pacientes operados por válvula aórtica bicúspide insuficiente

\begin{tabular}{|lccc|}
\hline Ecocardiografía & Preoperatoria & Postoperatoria & $\mathrm{p}$ \\
\hline Diámetro diastólico $(\mathrm{mm})$ & $64 \pm 5$ & $52,2 \pm 6,4$ & 0,015 \\
Diámetro sistólico (mm) & $40,7 \pm 2,9$ & $36 \pm 4,2$ & (ns) \\
Fracción de acortamiento (\%) & $35,6 \pm 3,5$ & $35 \pm 4,7$ & (ns) \\
\hline
\end{tabular}


sería más apropiado. Lo que caracteriza a estas válvulas, tanto del punto de vista anátomo-quirúrgico como ecocardiográfico, es su apertura transversal en "boca de pescado" o en "ojal".

La insuficiencia se produce por retracción de este velo fusionado o velo mayor, caso en que el reemplazo protésico es más apropiado, o por prolapso del mismo hacia la cavidad ventricular izquierda, lo que es susceptible de ser corregido conservando la válvula aórtica nativa, como se demuestra en esta experiencia y en otras publicadas en la literatura internacional ${ }^{10-13,25}$. Nuestra preferencia quirúrgica ha sido la resección triangular de la porción media prolapsante del velo fusionado, comprendiendo parte al menos del rafe medio. La base de resección debe ser de alrededor de $30 \%$ del borde libre del velo para mantener las dimensiones y flexibilidad de éste ${ }^{10,11,25}$. Ocasionalmente, en velos muy dúctiles, es posible efectuar sólo una plicatura del borde libre del velo ${ }^{13,25}$. Si bien éste es un procedimiento más rápido, también es estéticamente menos satisfactorio y su estabilidad en el tiempo aún no bien determinada. La anuloplastia subcomisural es un complemento a la resección triangular, cuya aplicación es una decisión intraoperatoria del cirujano, según su estimación de la continencia valvular luego de la resección triangular.

La válvula aórtica bicúspide es en la actualidad el principal sustrato anátomo-patológico para el desarrollo de una endocarditis infecciosa aórtica e igualmente es la principal causa de endocarditis bivalvular ${ }^{15,16,26,27}$. En esta serie, $22 \%$ de las válvulas presentaban signos de endocarditis, en 3 de ellas con perforación del velo no fusionado, lo que fue reparado con un parche de pericardio autólogo. Éste tiene claras ventajas sobre materiales sintéticos u otro tipo de tejidos biológicos, como el pericardio bovino.

La gran mayoría de los pacientes (62\%) no tuvo insuficiencia aórtica residual y el resto sólo una insuficiencia leve, en el ecocardiograma transesofágico intraoperatorio post reparación. Lo anterior es importante puesto que las evidencias actuales sugieren que grados mayores de insuficiencia se asocian a una elevada necesidad de reoperación ${ }^{12}$.

La insuficiencia mitral asociada con la válvula aórtica bicúspide insuficiente no es infrecuente, ya sea por dilatación del anillo mitral y retracción apical del aparato subvalvular por el crecimiento del ventrículo izquierdo o por secuelas de endocarditis mitral, como perforación de velos y/o rotura de cuerdas tendíneas. Ya que ambas situaciones mitrales son susceptibles de ser reparadas, no es infrecuente la reparación bivalvular, como en esta serie en que se efectuó en el 22\% de los casos $11,12,16,25$.

Finalmente, algunos de nuestros pacientes tuvieron una reparación de su válvula aórtica bicúspide insuficiente a través de una miniesternotomía ${ }^{28}$.

Morbimortalidad. Como en la mayoría de las series publicadas, en esta experiencia no hubo mortalidad ni morbilidad postoperatoria, lo que es importante de considerar si es que se plantea una intervención precoz de la insuficiencia aórtica, como lo sugieren estudios actuales ${ }^{10-13,24,25}$.

Seguimiento. La sobrevida de estos pacientes, y la calidad de ésta, ha sido excelente, al igual que en experiencias internacionales ${ }^{10-13,25}$. Lo anterior puede ser resultado de la juventud de la mayoría de ellos, así como de la menor necesidad de tratamiento anticoagulante.

Tres pacientes requirieron ser reoperados, en promedio a los 45 meses. Dos de ellos tenían una reparación mitral asociada por insuficiencia mitral funcional secundaria a dilatación y disfunción ventricular izquierda, lo que en la actualidad está claramente identificado como factor de riesgo de reoperación ${ }^{12,25}$. En ambos, la resección triangular se complementó con una anuloplastia subcomisural bilateral. El tercer paciente fue reoperado por una nueva endocarditis en el curso de una aplasia medular, por lo que estrictamente no debiera ser considerado una falla de la reparación. De hecho, este paciente volvió a presentar una endocarditis en la nueva prótesis aórtica, requiriendo un homoinjerto aórtico, para finalmente fallecer meses más tarde de su aplasia medular.

La probabilidad de estar libre de reoperación a 1 y 5 años, de $93 \%$ y $85 \%$ respectivamente, fue muy similar a la reportada por las clínicas Mayo y Cleveland y puede ser considerada satisfactoria ${ }^{10-12,25}$. Si bien un reemplazo protésico mecánico o biológico probablemente tendría una menor incidencia de reoperación a 5 años, esto sería a costa de complicaciones relacionadas con la prótesis, la que en el caso de las mecánicas es de $5 \%$ por año ${ }^{29}$. Igualmente, una reoperación 
de una prótesis biológica disfuncionante, lo que es más frecuente y precoz mientras más joven es el paciente, es técnicamente más demandante y probablemente de mayor riesgo que una reoperación por falla de una reparación aórtica ${ }^{30,31}$.

Todos los pacientes no reoperados estaban asintomáticos y los 3 que estaban en tratamiento anticoagulante tenían un procedimiento mitral asociado. En el seguimiento ecocardiográfico la mayoría de los pacientes no presentaban insuficiencia aórtica residual o solo una insuficiencia leve. Sin embargo, como en la experiencia de la Cleveland Clinic, 20\% de los pacientes presentaba una insuficiencia moderada, alguno de los cuales puede requerir una reoperación en el futuro, por lo que se mantienen en control ecocardiográfico y clínico estricto ${ }^{12}$.

$\mathrm{Si}$ bien el hecho de que no se encontrara una reducción estadísticamente significativa en el diámetro sistólico del ventrículo izquierdo, y por lo tanto tampoco en la fracción de acortamiento, puede ser explicado por el número limitado de pacientes, también podría traducir un daño, quizás irreversible del miocardio, con sustitución fibrosa, por la insuficiencia aórtica crónica, lo que podría apoyar una intervención quirúrgica cada

\section{REFERENCIAS}

1. Отто CM. Evaluation and management of chronic mitral regurgitation. N Engl J Med 2001; 345: 740-6.

2. Carpentier A. Cardiac valve surgery. The "French correction". J Thorac Cardiovasc Surg 1983; 86: 323-37.

3. Zalaquett R, Chamorro S, Braun S, Garrido L, Howard M, Morán S et al. Resultados alejados de la cirugía reconstructora de la insuficiencia mitral. Rev Méd Chile 1999; 127: 1093-100.

4. Zaiaquett R, Campiá C, Córdova S, Braun S, ChamoRRO G, IraRRázaval MJ, et al. Resultados alejados de la cirugía reparadora de la insuficiencia mitral degenerativa. Rev Méd Chile 2003; 131: 1355-64.

5. Jex RK, Schaff HV, Piehier JM, Orszulak ta, Puga FJ, KING RM, ET AL. Repair of ascending aortic dissection. Influence of associated aortic valve insufficiency on early and late results. J Thorac Cardiovasc Surg 1987; 93: 375-84. vez más precoz de estos pacientes, como es la tendencia en la actualidad ${ }^{24}$.

Limitaciones del estudio. Este es un estudio observacional descriptivo de un número limitado de pacientes y como tal tiene las limitaciones propias de éstos. Sin embargo, representa un grupo de pacientes, en su mayoría jóvenes, que se beneficiarán con un procedimiento que les permita conservar su propia válvula aórtica, si bien siempre bicúspide, funcional en cuanto a permitir un flujo unidireccional de sangre desde el ventrículo izquierdo a la aorta ascendente.

A pesar de las limitaciones señaladas, esta experiencia es concordante con la literatura internacional en cuanto a que la reparación de una válvula aórtica bicúspide tiene baja mortalidad y morbilidad operatoria y a un seguimiento promedio de casi 4 años una probabilidad de estar libre de reoperación aceptable, en especial si se considera el riesgo de complicaciones de las prótesis mecánicas y de falla estructural de las prótesis biológicas en pacientes jóvenes. Por lo mismo, la reparación de una válvula aórtica bicúspide insuficiente debe ser considerada una buena alternativa al reemplazo protésico mecánico y al xeno, homo o autoinjerto aórtico.

6. David TE, Armstrong S, Ivanov J, Feindel CM, OMRAN A, WeBв G. Results of aortic valve-sparing operations. J Thorac Cardiovasc Surg 2001; 122: 39-46.

7. Trusier GA, Wiшams WG, Smalhorn JF, Freedom RM. Late Results after repair of aortic insufficiency associated with ventricular septal defect. J Thorac Cardiovasc Surg 1992; 103: 276-81.

8. Rao V, Van Arsdell GS, David TE, Azakie A, WiшuMs WG. Aortic valve repair for adult congenital heart disease: a 22-year experience. Circulation 2000; 102 (supp III): III 40-3.

9. Schafers H, Fries R, Langer F, Nikoloudakis N, Graeter T, GRUNDMANN U. Valve-preserving replacement of the ascending aorta: remodeling versus reimplantation. J Thorac Cardiovasc Surg 1998; 116: 990-6.

10. Cosgrove DM, Rosenkranz ER, Hendren WG, BartLETT JC, STewart WJ. Valvuloplasty for aortic insufficiency. J Thorac Cardiovasc Surg 1991; 102: $571-6$. 
11. Fraser CD JR, Wang N, Mee RB, Lytle BW, McCARThy PM, SAPP SK ET AL. Repair of insufficient bicuspid aortic valves. Ann Thorac Surg 1994; 58: 386-90.

12. Casselman FP, Giwinov AM, Akhrass R, Kasirajan V, Biackstone EH, Cosgrove DM. Intermediate-term durability of bicuspide aortic valve repair for prolapsing leaflet. Eur J Cardiothorac Surg 1999; 15: 302-8.

13. Haydar HS, He GW, Hovaguimian H, McIrvin DM, KING DH, StaRR A. Valve repair for aortic insufficiency: surgical classification and techniques. Eur J Cardiothorac Surg 1997; 11: 258-65.

14. Morán S, Casanegra P, Maturana G, Dubernet J. Spontaneous rupture of a fenestrated aortic valve. J Thorac Cardiovasc Surg 1977; 73: 716-8.

15. Ferguson E, Reardon MJ, Letsou GU. The surgical management of bacterial valvular endocarditis. Curr Opin Cardiol 2000; 15: 82-5.

16. Zalaquett R, Garrido L, Casas F, Morán S, IrarRázaVAL MJ, BECKER P ET AL. Cirugía valvular reparadora en endocarditis infecciosa. Rev Méd Chile 2004; 132: 307-15.

17. Zalaquett R, Becker P, Irarrázaval MJ, Morán S, Maturana G, Navarro M et al. Cardioplegia retrógrada por canulación transauricular derecha del seno coronario. Rev Chil Cardiol 1993; 12: 68-71.

18. Edmunds LH, Ciark RE, Cohn LH, Miler DC, Weisel $\mathrm{RD}$. Guidelines for reporting morbidity and mortality after cardiac valvular operations. J Thorac Cardiovasc Surg 1988; 96: 351-3.

19. ZaLaQuetT R. Cirugía reparadora de la insuficiencia valvular mitral. Rev Chil Cir 1994; 46: 127-36.

20. Zalaquett R, Morán S, Irarrázaval MJ, Maturana G, Braun S, Chamorro G ET aL. Reconstrucción válvula mitral. Cirugía reparadora de la insuficiencia valvular mitral con evaluación ecocardiográfica transesofágica intraoperatoria. Rev Chil Cardiol 1996; 15: 78-84.

21. Fenoglo JJ Jr, McAwster HA Jr, DeCastro CM, Davia JE, Cheitun MD. Congenital bicuspid aortic valve after age 20. Am J Cardiol 1977; 39: 164-9.
22. Fedak PW, Verma S, David TE, Lfask RL, Weisel RD, BUTANY J. Clinical and pathophysiological implications of a bicuspid aortic valve. Circulation 2002; 20: 106: 900-4.

23. Mils $P$, ieech G, Davies M, Leathan A. The natural history of a non-stenotic bicuspid aortic valve. $\mathrm{Br}$ Heart J 1978; 40: 951-7.

24. Dujardin KS, Enríquez-Sarano M, Schaff HV, Bailey KR, SEward JB, TAJIK AJ. Mortality and morbidity of aortic regurgitation in clinical practice. A long-term follow-up study. Circulation 1999; 13: 99: 1851-7.

25. Minakata K, Hartzell S, Kenton Z, Dearani J, Richard D, Orszulak T et al. Is repair of aortic valve regurgitation a safe altemative to valve replacement? J Thorac Cardiovasc Surg 2004; 127: 645-53.

26. Mylonakis E, CaLderwood SB. Infective endocarditis in adults. N Engl J Med 2001; 345: 1318-30.

27. Guimnov AM, Díaz R, Biackstone EH, Pettersson GB, Sabick JF, Lytie BW et al. Double Valve Endocarditis. Ann Thorac Surg 2001; 71: 1874-9.

28. Zalaquett R, Baeza C, Irarrázaval MJ, Morán S, Becker P, Maturana G et al. Mini esternotomía en cirugía valvular. Rev Chil Cardiol 1999; 18: 63-8.

29. Khan SS, Trento A, DeRobertis M, Kass RM, Sandhu M, CZER LS ET AL. Twenty-year comparison of tissue and mechanical valve replacement. J Thorac Cardiovasc Surg 2001; 122: 257-69.

30. FAnN JI, Miler DC, Moore KA, Mitchell RS, Oyer PE, Stinson EB ET AL. Twenty-year clinical experience with porcine bioprostheses. Ann Thorac Surg 1996; 62: 1301-11.

31. Jamieson WR, Ling H, BurR LH, Fradet GJ, MiyagisHIMA RT, Janusz MT et AL. Carpentier-Edwards supraannular porcine bioprothesis evaluation over 15 years. Ann Thorac Surg 1998; 66: S49-52.

Agradecimientos:

Los autores expresan su sincero agradecimiento a la Srta. Bioestadística Alessandra Gederlini y a su secretaria Srta. Verónica Delgado, por su asistencia estadística y por las múltiples correcciones del manuscrito. 